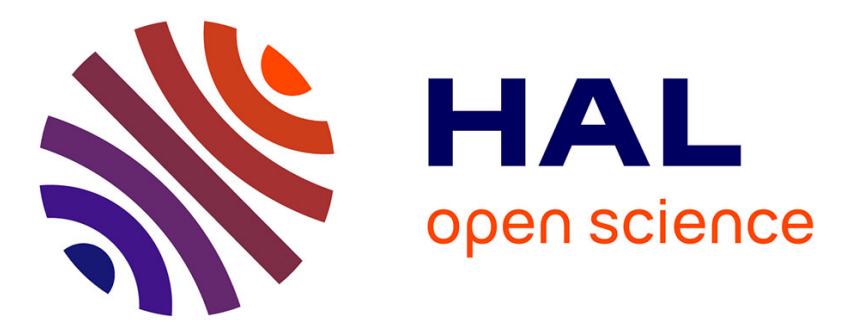

\title{
Computationally Assisted Mechanistic Investigation into Hypervalent Iodine Catalysis: Cyclization of $\mathrm{N}$ -Allylbenzamide
}

Smaher Butt, Mirdyul Das, Jean-Marc Sotiropoulos, Wesley Moran

\section{- To cite this version:}

Smaher Butt, Mirdyul Das, Jean-Marc Sotiropoulos, Wesley Moran. Computationally Assisted Mechanistic Investigation into Hypervalent Iodine Catalysis: Cyclization of N -Allylbenzamide. Journal of Organic Chemistry, 2019, 84 (23), pp.15605-15613. 10.1021/acs.joc.9b02623 • hal-02378237

\section{HAL Id: hal-02378237 \\ https://hal.science/hal-02378237}

Submitted on 20 Nov 2020

HAL is a multi-disciplinary open access archive for the deposit and dissemination of scientific research documents, whether they are published or not. The documents may come from teaching and research institutions in France or abroad, or from public or private research centers.
L'archive ouverte pluridisciplinaire HAL, est destinée au dépôt et à la diffusion de documents scientifiques de niveau recherche, publiés ou non, émanant des établissements d'enseignement et de recherche français ou étrangers, des laboratoires publics ou privés. 


\section{Computationally Assisted Mechanistic Investigation}

\section{into Hypervalent Iodine Catalysis: Cyclization of $N$ -}

\section{Allylbenzamide}

Smaher E. Butt," Mirdyul Das, "Jean-Marc Sotiropoulos ${ }^{\S}$ and Wesley J. Moran*"

" Department of Chemistry, University of Huddersfield, Queensgate, Huddersfield HD1 3DH, U.K.

$\S$ Université de Pau et des Pays de l'Adour, IPREM (CNRS-UMR 5254), Technopole Hélioparc, 2 Avenue du Président Pierre Angot, 64053 Pau Cedex 09, France

w.j.moran@hud.ac.uk

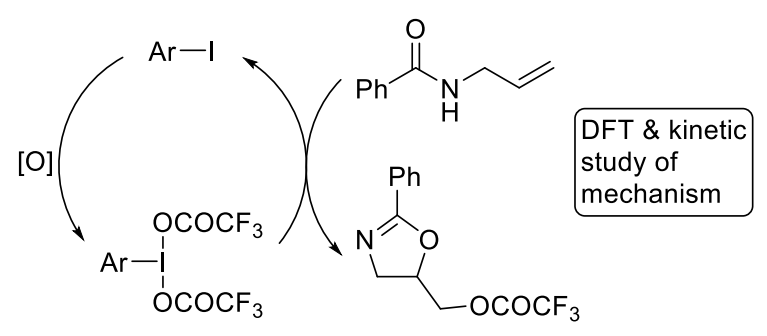

$\Delta \mathrm{G}^{\ddagger}$ of cyclization correlates with Arl oxidation potential

BUT, most easily formed $\mathrm{Arl}\left(\mathrm{OCOCF}_{3}\right)_{2}$ decompose rapidly

ABSTRACT: Previous experimental work identified 2-iodoanisole as the best precatalyst for the oxidative cyclization of $\mathrm{N}$-alkenylamides into 2-oxazolines. Herein, we describe our investigation into the effect on reaction rate based on the structure of the iodoarene precatalyst. We also reveal 
the mechanism of the cyclisation based on DFT modelling and obtain a clear correlation between observed reaction rates and computationally derived activation energies for different iodoarenes. In addition, the rate-limiting step is shown to be the cyclization of the substrate which is zero order in the concentration of the iodoarene precatalyst. The rate of the cyclization is found to correlate with the ease of oxidation of the iodoarene, however the most easily oxidized iodoarenes generate iodine(III) species that decompose readily. Finally, loss of iodoarene from the cyclized intermediate can proceed by either ligand-coupling or $\mathrm{S}_{\mathrm{N}} 2$ displacement (reductive elimination), and this is shown to be substrate dependent.

\section{INTRODUCTION}

Hypervalent iodine chemistry has continued to receive considerable attention from synthetic practitioners over the past decades with a wide variety of useful reactivities revealed. ${ }^{1}$ Hypervalent iodine compounds can possess reactivity profiles comparable to some heavy metal and transition metal complexes but may not suffer from disadvantages such as toxicity, cost or scarcity. In particular, the ability to access reactive $\lambda^{3}$-iodanes in-situ from iodoarenes and an oxidant has permitted the discovery of a range of reactions that are catalytic in iodoarene. ${ }^{2}$ This is especially important as the preparation and isolation of $\lambda^{3}$-iodanes is problematic in many cases and often requires considerable experimental investigation to identify suitable conditions. ${ }^{3}$ As such, the insitu generation and reaction of $\lambda^{3}$-iodanes can be advantageous as isolation is avoided and, of course, allows catalytic applications.

Fuchigami reported in 1994 that 4-iodoanisole can be used in as little as $5 \mathrm{~mol} \%$ to catalyze the

gem-difluorination of dithioketals in an electrochemical cell. ${ }^{4}$ However, the first examples of 
iodoarene catalysis are often ascribed to the two groups of Ochiai and Kita who simultaneously published their studies in 2005. Ochiai described the iodobenzene-catalyzed $\alpha$-acetoxylation of ketones employing $m$-chloroperbenzoic acid as the stoichiometric oxidant. ${ }^{5}$ Four other iodoarene precatalysts were investigated in this study but these were all found to give similar or inferior yields of product $(67 \%$ with $p$-methoxy-, $84 \%$ with $p$-methyl-, $81 \%$ with $p$-chloro-, and $58 \%$ with $p$-nitroiodobenzene); however, there is no mention of the effect on the rate of the reaction. Kita and co-workers reported the spirocyclization of phenols using as little as $1 \mathrm{~mol} \%$ of hypervalent iodine reagent with $m$-chloroperbenzoic acid as the re-oxidant. ${ }^{6}$ Later, $\mathrm{Li}$ and co-workers investigated the syn-diacetoxylation of alkenes catalyzed by six different iodoarenes and discovered that iodomesitylene provided the highest yields and diastereoselectivities (Scheme 1a). ${ }^{7}$ Similarly, Zhdankin demonstrated that 5 -iodo- $m$-xylene was superior to four other iodoarenes in the conversion of oximes into nitrile oxides. ${ }^{8}$ Murphy described an iodoarene-catalyzed intramolecular alkene arylation strategy to access polycyclic aromatic hydrocarbons which was superior with 4-iodotoluene rather than iodobenzene, 4-iodoanisole or 1-iodo-4-nitrobenzene (Scheme 1b) ${ }^{9}$ 
Scheme 1. Effect of iodoarene substitution on reaction outcomes

(a) Li, 2012

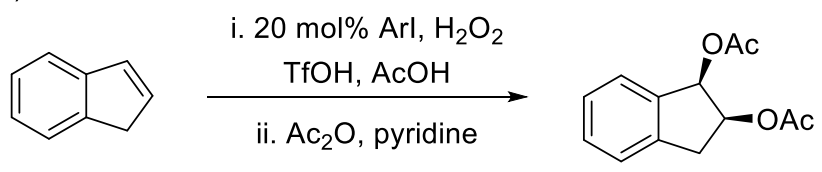

Arl: Phl (82\%, 12.5:1 dr); 4-ClC ${ }_{6} \mathrm{H}_{4} \mathrm{I}(81 \%, 5.3: 1 \mathrm{dr}) ; 4-$ $\mathrm{MeOC}_{6} \mathrm{H}_{4} \mathrm{I}(85 \%, 6.7: 1 \mathrm{dr}) ; 2,4,6-\mathrm{Me}_{3} \mathrm{C}_{6} \mathrm{H}_{2} \mathrm{I}(84 \%, 14: 1 \mathrm{dr})$

(b) Murphy, 2018

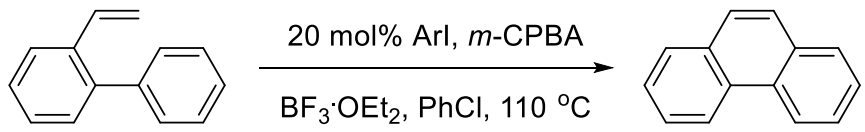

Arl: Phl (63\%); 4- $\mathrm{NO}_{2} \mathrm{C}_{6} \mathrm{H}_{4} \mathrm{I}(48 \%) ; 4-\mathrm{MeOC}_{6} \mathrm{H}_{4} \mathrm{l}(70 \%)$;

$4-\mathrm{MeC}_{6} \mathrm{H}_{4} \mathrm{l}(79 \%)$

(c) Our work, 2015
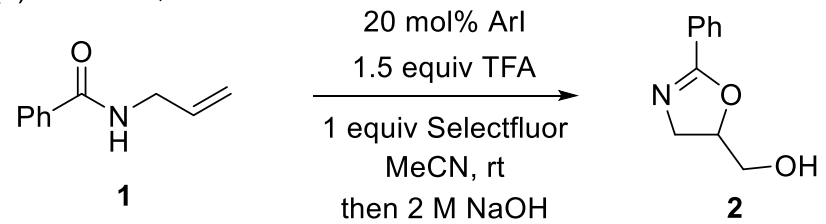

Arl: $\mathrm{Phl}(6 \%), 2-\mathrm{MeOC}_{6} \mathrm{H}_{4} \mathrm{l}(62 \%) ; 3-\mathrm{MeOC}_{6} \mathrm{H}_{4} \mathrm{I}(9 \%)$;

$4-\mathrm{MeOC}_{6} \mathrm{H}_{4} \mathrm{I}(10 \%) ; 3,5-\mathrm{Me}_{2} \mathrm{C}_{6} \mathrm{H}_{3} \mathrm{I}(13 \%)$

In this regard, we reported that 2-iodoanisole is a superior precatalyst for the oxidative cyclization of $\mathrm{N}$-alkenylamides such as $\mathbf{1}$ into 2-oxazolines $\mathbf{2}$ compared to several other iodoarenes (Scheme 1c). ${ }^{10} \mathrm{We}$ also discovered that the cyclization of $N$-propargyl amides and $\beta$-amidoketones performed better with 2-iodoanisole as the precatalyst compared to iodobenzene. ${ }^{11}$

We were interested in investigating the mechanism of our cyclization of $N$-alkenylamides using kinetic and computational techniques, especially as relatively few such studies of hypervalent iodine reactions have been reported to date. ${ }^{12,13}$ Of crucial importance to our study, was the determination of the reasons behind the different reaction outcomes with iodoarene precatalyst variation.

Houk, Xue and co-workers recently published a computational study on the mechanism and origins of chemo- and stereoselectivities in the chiral iodoarene-catalyzed difluorination of 
styrenes. ${ }^{14}$ This report is a tour de force in rationalizing the reasons for enantioselectivies in the difluorination reaction but does not consider the initial iodoarene oxidation. Indeed, the authors state that "the overall activation free energy of $18.9 \mathrm{kcal} / \mathrm{mol}$ is not consistent with the $60 \mathrm{~h}$ reaction times required experimentally." Studying the early part of the reaction mechanism is critical for our study as we are interested in understanding the generation and catalytic properties of the hypervalent iodine species involved.

\section{RESULTS AND DISCUSSION}

Model Reaction, Initial Rate Data and Proposed Catalytic Cycle. We initiated our study by investigating the rate of cyclization of $N$-allylbenzamide 1 using different iodoarene precatalysts under our standard reaction conditions of 1.5 equivalents of Selectfluor as oxidant, 1.5 equivalents of trifluoroacetic acid (TFA) in acetonitrile at room temperature, $\sim 20^{\circ} \mathrm{C}$ (Scheme 2). We saw a clear and substantial difference in initial rates between different precatalysts as well as marked variation in final yields of products. With iodobenzene $\mathbf{4}$ as precatalyst, the initial rate was just $5.16 \times 10^{-6} \mathrm{~s}^{-1}$ meaning that $50 \%$ conversion of starting material took 52 hours. Employing 2-iodotoluene 5 led to a somewhat faster reaction; however, the presence of the electron-withdrawing $p$-nitro group in $\mathbf{6}$ dramatically reduced the rate. The electron-donating $o$ methoxy and $p$-methoxy groups in 2-iodoanisole 7 and 4-iodoanisole 8 respectively led to significantly increased rates. The presence of the second $o$-methoxy substituents in $\mathbf{9}$ had a detrimental effect on the rate of cyclization compared to 7 , which was somewhat countered by the ester in the para-position of iodoarene 10. Most important from a synthetic standpoint is that the use of 2-iodoanisole led to the highest yield of oxazoline 2 after purification, in accord with our previous observations. 
Scheme 2. Initial rates for conversion of $N$-allylbenzamide 1 into oxazoline 3 with iodoarene precatalysts 4-10, determined by ${ }^{1} \mathrm{H}$ NMR analysis

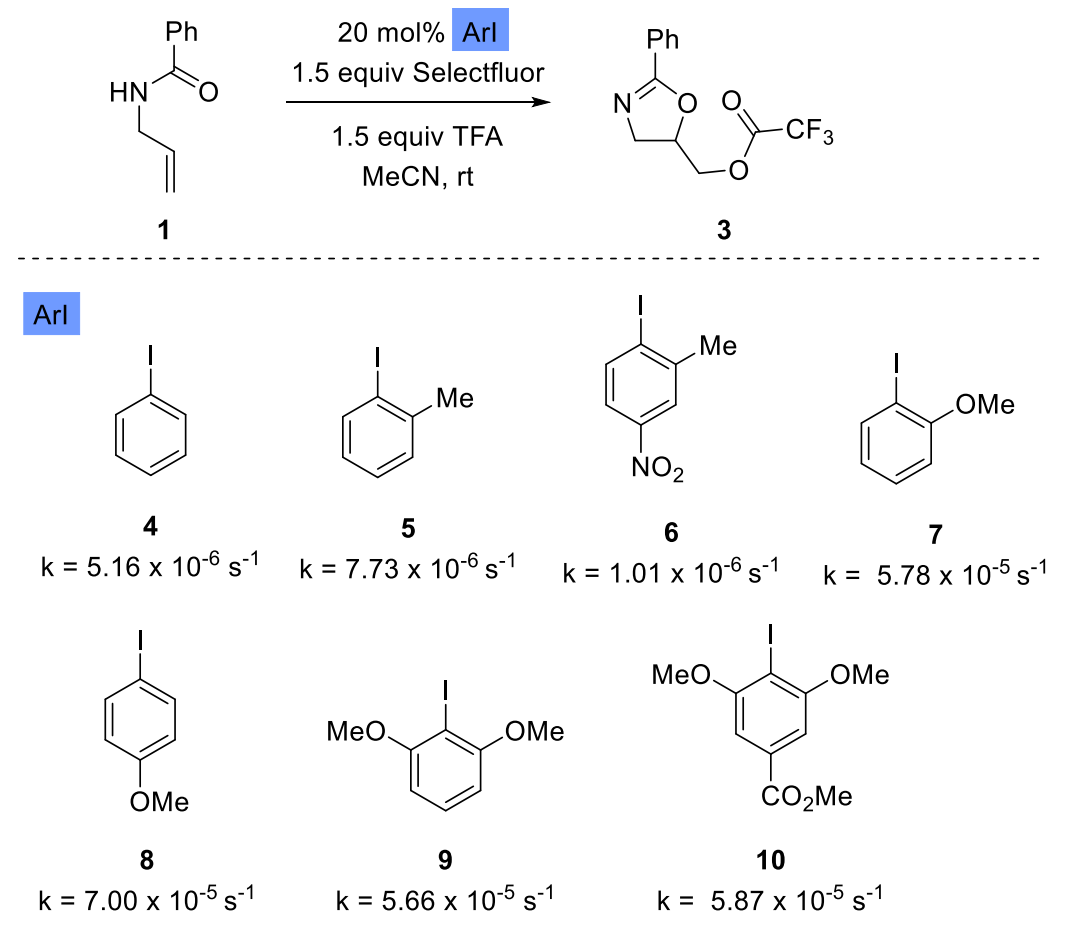

In order to determine the order of the reaction with respect to the iodoarene concentration, we carried out the Variable Time Normalization Analysis (VTNA), which involved changing the initial quantity of 2-iodoanisole 7 and monitoring the rate of reaction by NMR analysis. ${ }^{15}$ The profiles of reactions performed with different catalyst loadings are plotted on a normalized time scale, $t[\text { cat }]_{\mathrm{T}}{ }^{n}$, against the concentration of the substrate 1 . The best fit of the data led to the conclusion that the reaction is zero order in 2-iodoanisole 7 (Scheme 3). This unusual finding is in accord with our previous experimental findings during reaction optimization but it does not help to rationalize the relatively high catalyst loadings required for acceptable yields of products. A reaction which is zero order in catalyst is one where the catalyst is fully saturated in substrate(s) and this is more common in photochemical reactions, ${ }^{16}$ or heterogeneous catalysis. ${ }^{17}$ 
In our case, this finding can be rationalized by consideration of the NMR spectra obtained during the kinetic experiments, as only the iodoarenes were observed in their initial concentrations. That is, no $\lambda^{3}$-iodanes were observed by NMR analysis during the course of the reactions. This suggested that the concentration of $\lambda^{3}$-iodane in the reaction mixture was very low and upon formation by oxidation of an iodoarene, the $\lambda^{3}$-iodane initiated the reaction sequence. In addition, $\lambda^{3}$-iodanes tend to have low solubility (especially compared to the precursor iodoarenes), therefore the reaction could be occurring in a heterogeneous fashion; indeed, precipitation occurs during the course of these reactions.

Scheme 3. Determination of reaction order with respect to concentration of 2 -iodoanisole 7

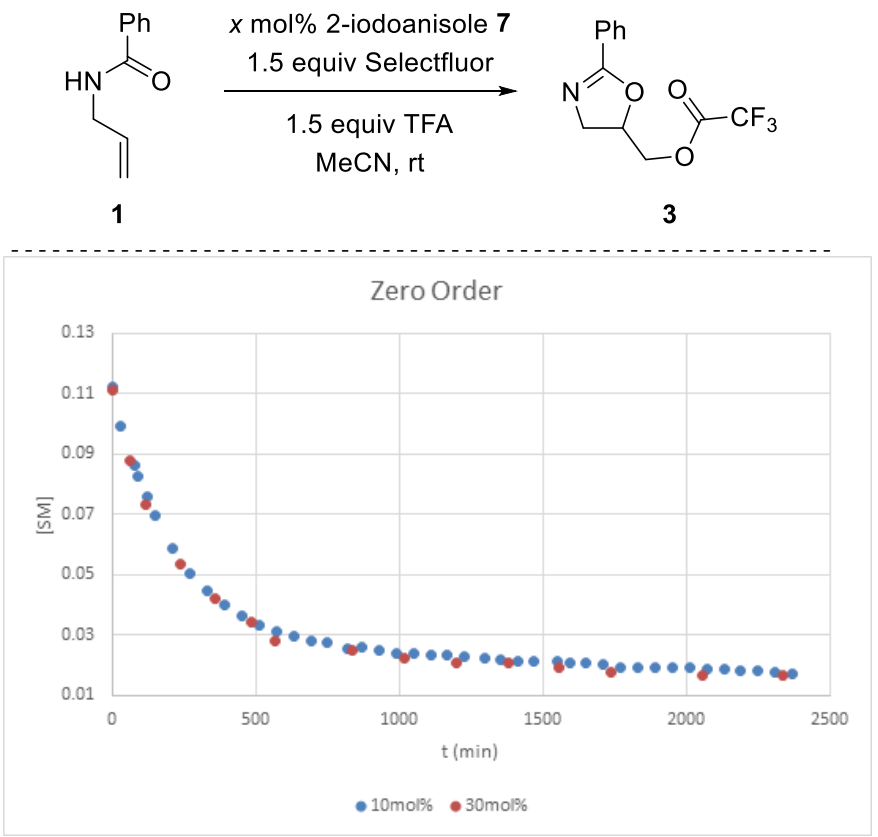

The mechanism proposed in the original study involved oxidation of the iodoarene precatalyst to the iodine(III) species, which coordinated to the alkene of $N$-allylbenzamide $\mathbf{1}$ and activated it to intramolecular attack by the amide oxygen forming intermediate $\mathbf{1 1}$ (Scheme 4). Loss of a proton and displacement of the iodonium by trifluoroacetate formed the final product $\mathbf{3}$. It was found 
that some of the trifluoroacetate esters $\mathbf{3}$ were unstable to chromatographic purification, therefore saponification to the alcohol 2 was performed during workup. It was anticipated that trifluoroacetate would be bound to the iodine(III) center during the course of the reaction, but fluoride was also present in solution. Similar reaction mechanisms have been proposed for other cyclizations of alkene substrates with hypervalent iodine compounds. ${ }^{18}$

Scheme 4. Proposed mechanism for the iodoarene-catalyzed cyclization of $N$-allylbenzamides 1

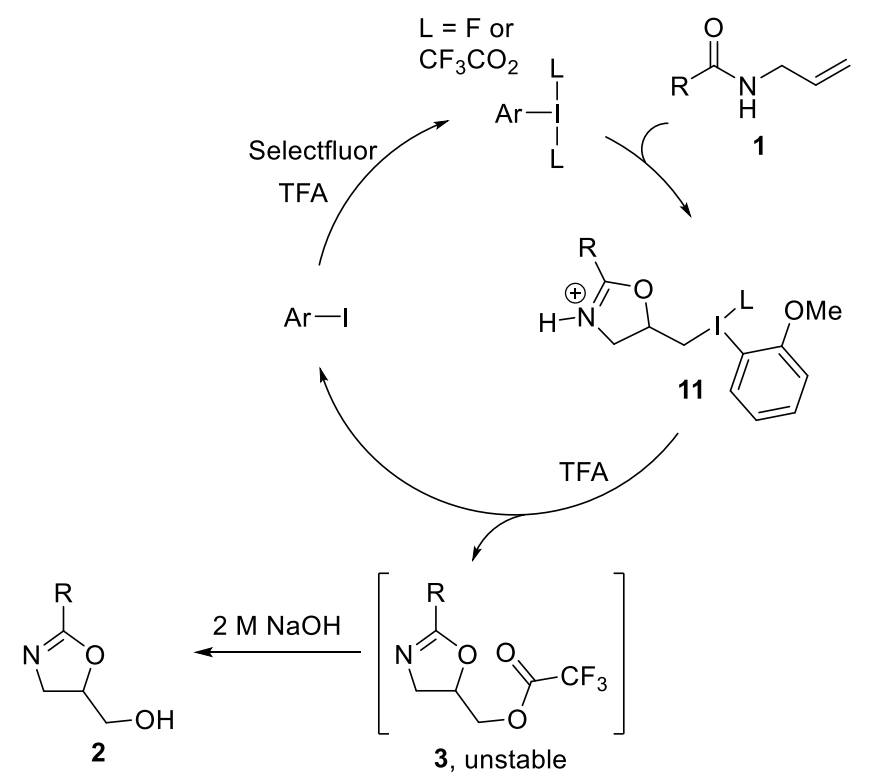

At this point we decided to study the reaction mechanism computationally to help rationalize the differences in rates observed with different iodoarenes precatalysts with the intention to use the knowledge gained to identify superior precatalysts.

Mechanism of Iodoarene-Catalyzed Cyclization of $\mathrm{N}$-allylbenzamide 1 . We began our modelling of the reaction mechanism by ignoring the initial oxidation of the iodoarene, like other published studies, and started with bis(trifluoroacetoxy)iodobenzene 12, which should be formed under the reaction conditions (Figure 1). Loss of a trifluoroacetate ligand from $\mathbf{1 2}$ to form $\mathbf{1 3}$ 
followed by formation of activated alkene complex 14, i.e. a dissociative mechanism, was found to be possible but of high energy. In contrast, an associative mechanism whereby a trifluoroacetate ligand formed a hydrogen-bond with the incoming amide and underwent a concerted rotation-coordination process was of lower energy, i.e. formation of intermediate $\mathbf{1 5 a}$ via TS[14-15a]. However, the barrier was further reduced to $21.3 \mathrm{kcal} \mathrm{mol}^{-1}$ when an extra molecule of trifluoroacetic acid was involved in the alkene activation i.e., TS[14-15b], leading to the intermediate 15b. Cyclization of $\mathbf{1 5 b}$ (and 15a) proceeded through $\mathbf{T S}[\mathbf{1 5 - 1 6}]$, which was higher in energy by $4.7 \mathrm{kcal} \mathrm{mol}^{-1}$, to form iodonium 16. Loss of a proton formed oxazoline 17 which was then converted to the final product 3 with loss of iodobenzene 4 . Two possible mechanisms were envisaged here: ligand coupling and $\mathrm{S}_{\mathrm{N}} 2$ displacement, the latter of which can also be termed reductive elimination. Modelling revealed that the $\mathrm{S}_{\mathrm{N}} 2$ process would proceed through TS[17-3]S which is $5 \mathrm{kcal} \mathrm{mol}^{-1}$ lower in energy than the corresponding ligand coupling transition state TS[17-3]LC. Attempts to model the reaction mechanism with $\lambda^{3}$-iodanes bearing fluoride ligands were unsuccessful. ${ }^{19}$ Attempts to find mechanistic pathways involving cisaddition to the alkene were also not fruitful. 

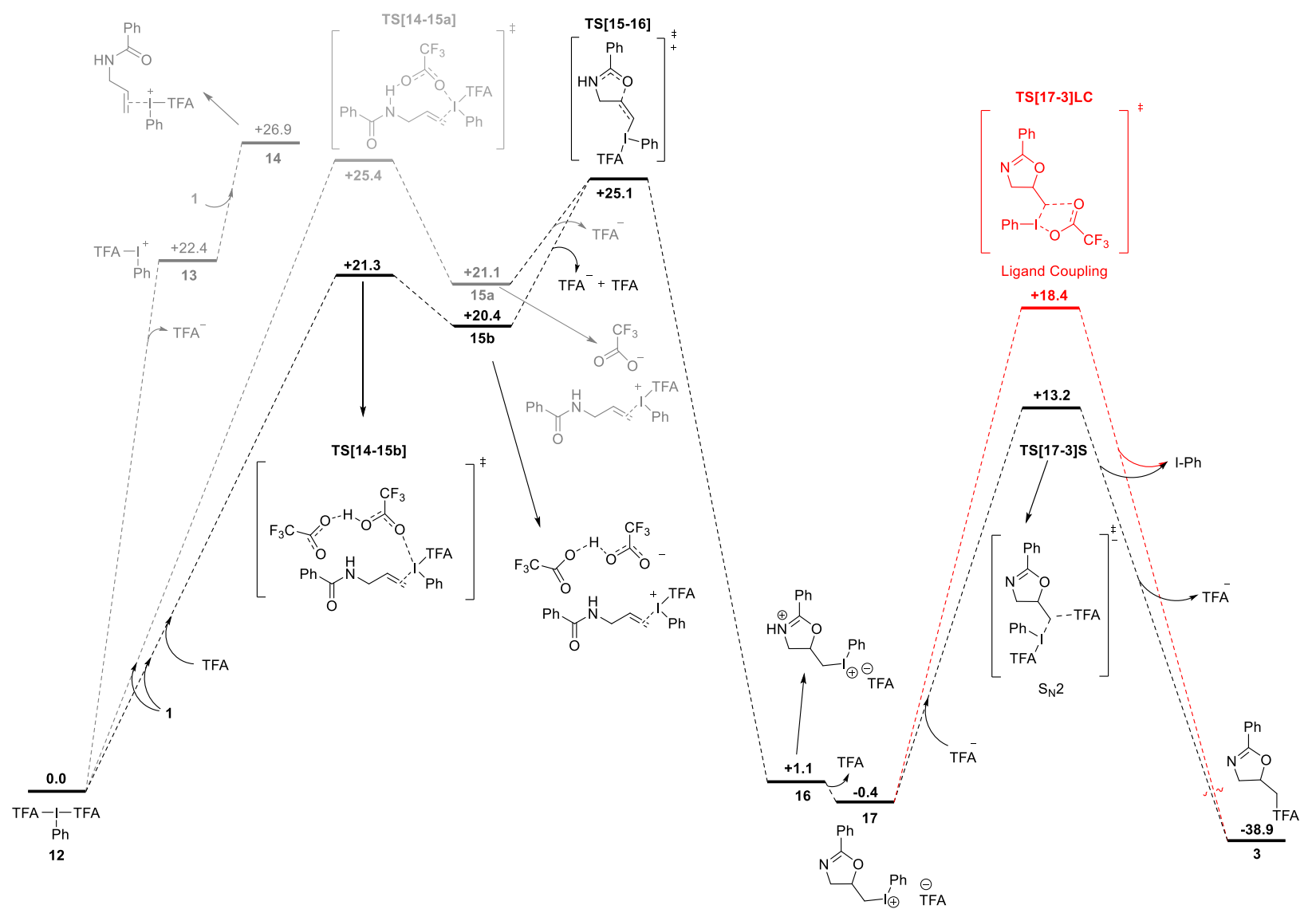

Figure 1. Calculated potential energy profile for the $\mathrm{PhI}\left(\mathrm{O}_{2} \mathrm{CCF}_{3}\right)_{2}$ 12-mediated cyclization of $\mathrm{N}$ allylbenzamide 1. Free energies are reported in $\mathrm{kcal} \mathrm{mol}^{-1}$.

The optimized geometries of all structures in the calculated mechanism conformed to our expectations with all bond lengths and angles being as anticipated (Figure 2). Importantly, the whole mechanistic pathway has been connected by IRC (intrinsic reaction coordinate) calculations (see SI for details). 


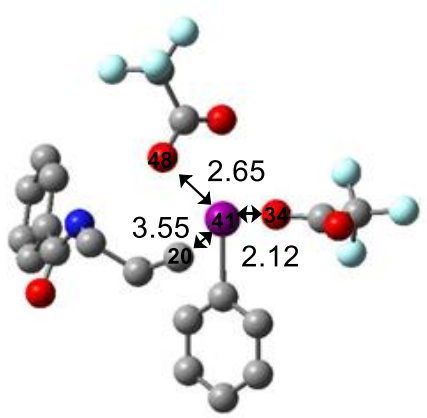

TS[14-15a]

O48-141-O34 $=136.4^{\circ}$ $\mathrm{C} 20-141-\mathrm{O} 34=153.6^{\circ}$

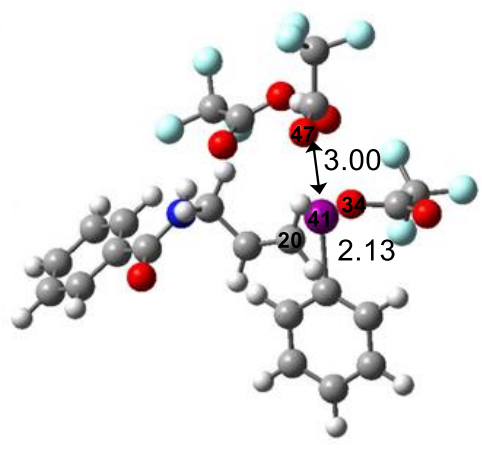

15b

$\mathrm{O} 47-141-\mathrm{O} 34=118.8^{\circ}$ $\mathrm{C} 20-141-\mathrm{O} 34=173.6^{\circ}$

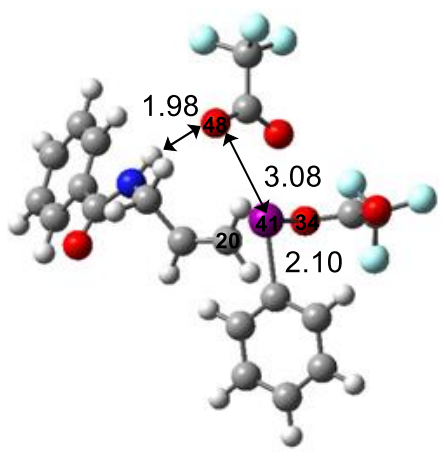

$15 a$

O48-141-034 $=114.5^{\circ}$

$\mathrm{C} 20-141-\mathrm{O} 34=165.3^{\circ}$

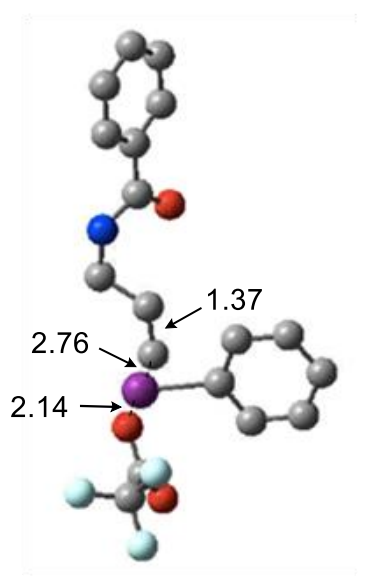

TS[15-16]

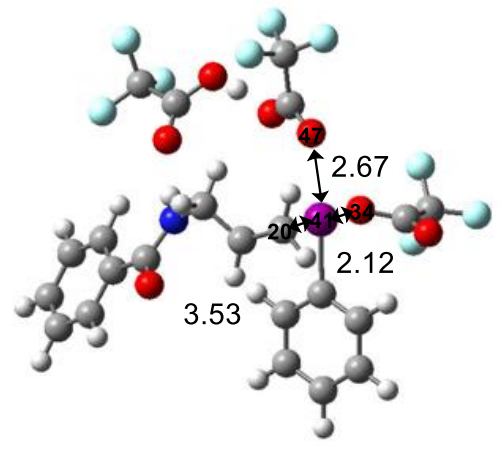

TS[14-15b]

$\mathrm{O} 47-141-\mathrm{O} 34=137.8^{\circ}$

$\mathrm{C} 20-141-\mathrm{O} 34=153.5^{\circ}$

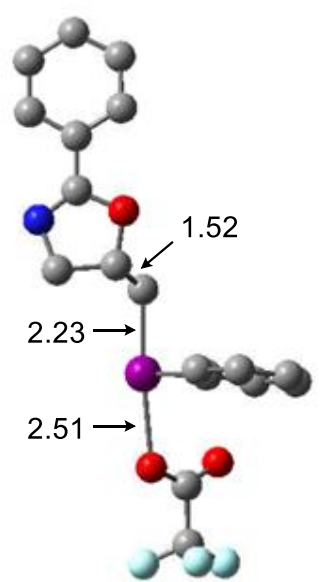

17

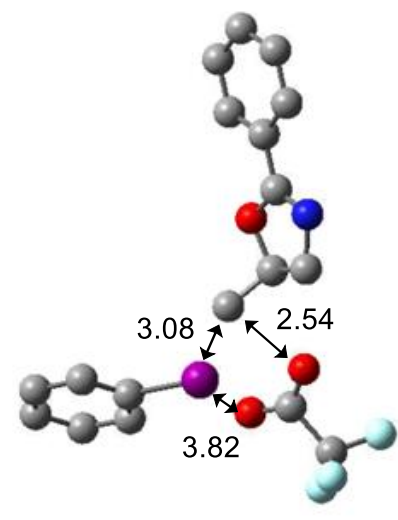

TS[17-3]LC

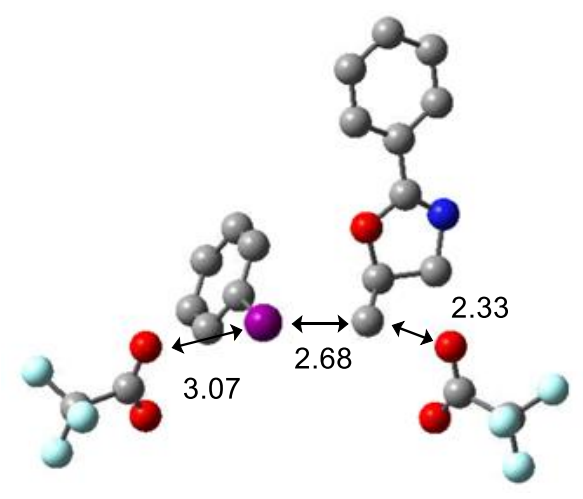

TS[17-3]S 
Figure 2. Calculated geometries of intermediates and transition structures of the $\mathrm{PhI}\left(\mathrm{O}_{2} \mathrm{CCF}_{3}\right)_{2}-$ mediated cyclization of $N$-allylbenzamide 1 . Some hydrogen atoms are omitted for clarity.

Comparing and Contrasting Different Iodoarene Precatalysts. The rate of cyclization of $\mathrm{N}$ allylbenzamide catalyzed by iodobenzene is slow at room temperature and the calculated value of $25.1 \mathrm{kcal} \mathrm{mol}^{-1}$ for $\mathbf{T S}[\mathbf{1 5 - 1 6}]$ is high for a room temperature process. At this point, we decided to model the transition state of the rate-limiting step with all of the iodoarenes studied in our initial kinetic work (assuming no change in mechanism or rate-limiting step).

The modelling of the TS[15-16] transition state for each iodoarene proceeded as expected, however the presence of a substituent in the 2-position led to two diastereomeric transition states. For example, with 2-iodoanisole the two diastereomeric transition states $\mathbf{1 8 a}$ and $\mathbf{1 8 b}$ had an energy difference of $1.3 \mathrm{kcal} \mathrm{mol}^{-1}$ (Figure 3). 


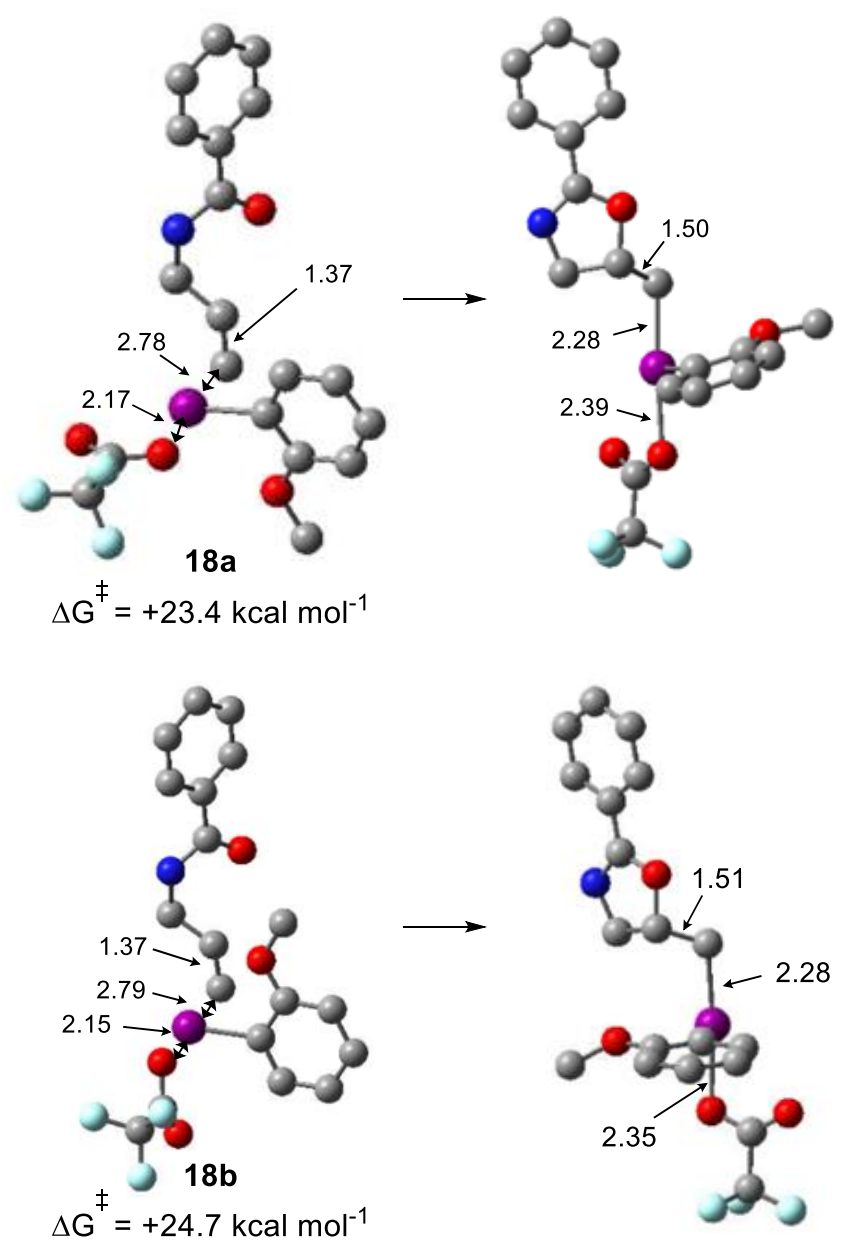

Figure 3. Calculated geometries of two diastereomeric transition states $\mathbf{1 8 a}$ and $\mathbf{1 8 b}$ and the subsequent cyclized intermediates derived from 2-iodoanisole.

Comparing the experimentally determined initial rates with the computationally derived free energy values for the rate-limiting step showed a good match for all of the iodoarenes studied (Table 1). The data suggested that installing an electron-withdrawing group para to the iodine led to a raising of the activation energy and a lowering of the rate (entry 3 ) whereas an electrondonating group in the ortho or para position lowered the activation energy and increased the rate (entries 4 and 5). Interestingly, having two ortho-methoxy groups did not lead to an additive effect and the activation energy was similar to having a single methoxy group in the ortho or 
para position (entry 6 versus entries 4 and 5). The only outlier was iodoarene $\mathbf{1 0}$ which performed worse than anticipated from its calculated value (entry 7).

Table 1. Comparison of experimental rate data with calculated free energies of rate-limiting step with different iodoarenes

\begin{tabular}{|c|c|c|c|}
\hline entry & ArI & $\mathrm{k} / \mathrm{s}^{-1}$ & $\begin{array}{l}\Delta \mathrm{G}_{\text {calc }}^{\ddagger} / \\
\mathrm{kcal} \mathrm{mol}^{-1}\end{array}$ \\
\hline 1 & iodobenzene 4 & $5.16 \times 10^{-6}$ & 25.1 \\
\hline 2 & 2-iodotoluene 5 & $7.73 \times 10^{-6}$ & 25.9 \\
\hline 3 & $\begin{array}{l}\text { 2-iodo-5- } \\
\text { nitrotoluene } 6\end{array}$ & $1.01 \times 10^{-6}$ & 26.0 \\
\hline 4 & 2-iodoanisole 7 & $5.78 \times 10^{-5}$ & 23.4 \\
\hline 5 & 4-iodoanisole 8 & $7.00 \times 10^{-5}$ & 23.2 \\
\hline 6 & $\begin{array}{l}\text { 2-iodo-1,3- } \\
\text { dimethoxybenzene } \\
\mathbf{9}\end{array}$ & $5.66 \times 10^{-5}$ & 23.5 \\
\hline 7 & $\begin{array}{l}\text { methyl 4-iodo-3,5- } \\
\text { dimethoxybenzoate } \\
\mathbf{1 0}\end{array}$ & $5.87 \times 10^{-5}$ & 21.9 \\
\hline
\end{tabular}

Oxidation of Iodoarenes. Computing transition state energies is computationally demanding, therefore we sought a simple, fast method to predict iodoarene structures with lower TS[15-16] transition state energies than 2-iodoanisole. The oxidation of the iodoarene to the $\lambda^{3}$-iodane had been ignored up to this point in our computations as it was presumed to be complex with several discrete mechanistic steps. However, our hypothesis was that more easily oxidized iodoarenes correlated with lower transition state energies and faster reaction rates. Accordingly, the oxidation 
potential was computed for our studied iodoarenes using the method described by Nicewicz and coworkers. ${ }^{20}$ These values were compared with the experimental rate values and a clear correlation was observed, confirming our hypothesis (Figure 4). In addition, the experimental rate values were shown to be proportional to the corresponding calculated HOMO energies of the iodoarenes.

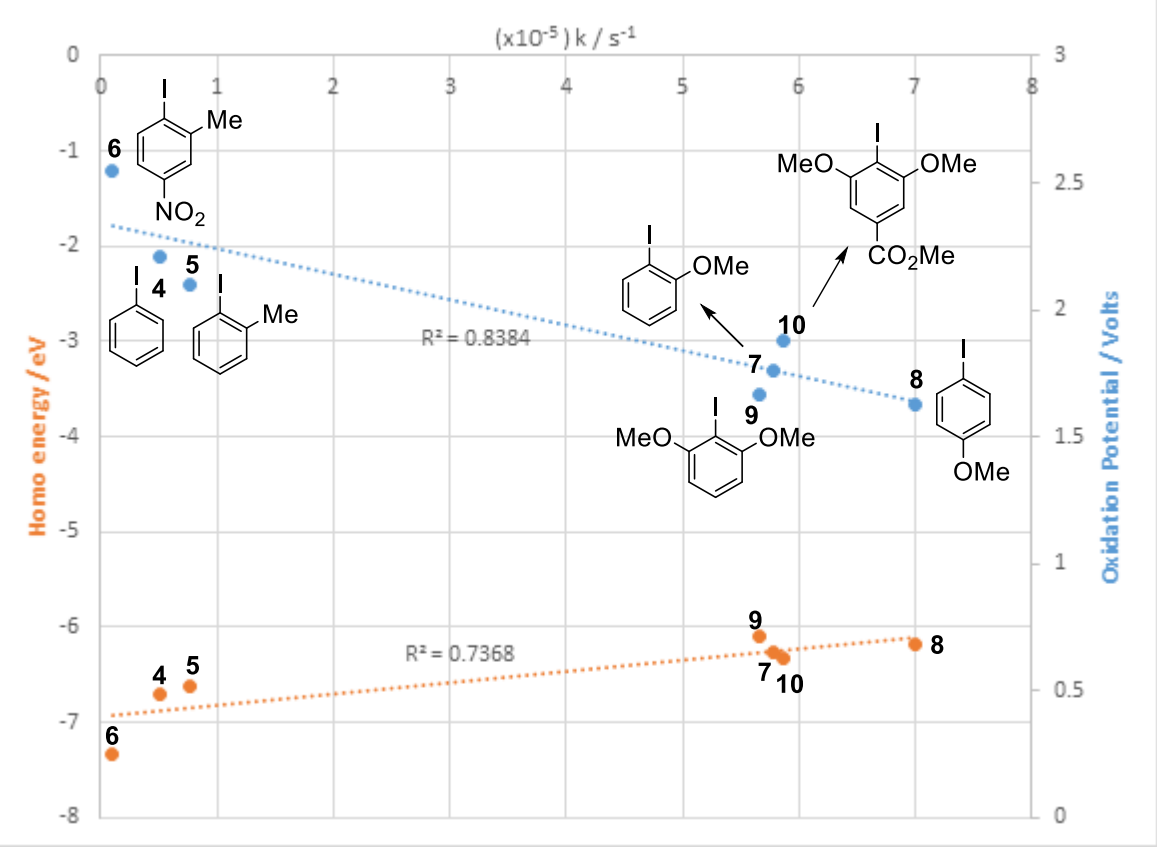

Figure 4. Correlation between calculated oxidation potentials of iodoarenes and experimental rate data derived free energies of TS[15-16]

With this correlation confirmed, the identities of iodoarenes with lower oxidation potentials were sought as these were envisaged to lead to superior catalysts. 1-Iodo-2,4-dimethoxybenzene 19 and 2-iodo-1,3,5-trimethoxybenzene $\mathbf{2 0}$ were quickly identified as being more easily oxidized and their oxidation potentials were calculated to be $1.40 \mathrm{~V}$ and $1.34 \mathrm{~V}$ respectively. Unfortunately, kinetic experiments with these precatalysts in our cyclization reaction indicated that the reactions were somewhat slower than when using 2-iodoanisole 7 as the precatalyst and, crucially, the yields of product were only $20-30 \%$. Furthermore, the iodoarenes $\mathbf{1 9}$ and $\mathbf{2 0}$ disappeared during the course of the reactions. 
In order to probe the oxidation of the iodoarenes, the rate of oxidation by Selectfluor was monitored by ${ }^{1} \mathrm{H}$ NMR analysis (Scheme 5). Iodobenzene 4, 2-iodotoluene 5 and ester 10 required about 24 hours to reach full conversion to the $\lambda^{3}$-iodane (entries 1, 2 and 3). 2-Iodoanisole 7 was fully oxidized in about 14 hours (entry 4) and 4-iodoanisole 8 was slightly quicker (entry 5). However, 1-iodo-2,4-dimethoxybenzene $\mathbf{1 9}$ and 2-iodo-1,3,5-trimethoxybenzene $\mathbf{2 0}$ were completely consumed by the oxidant but no products were evident by NMR analysis (entries 6 and 7). It is known that 1,3,5-trimethoxybenzene decomposes under electrolysis in aqueous sulfuric acid to generate carboxylic acids, carbon dioxide and water. ${ }^{21}$ It appears that iodoarenes $\mathbf{1 9}$ and $\mathbf{2 0}$ undergo a similar decomposition process under our reaction conditions on a slightly faster time scale to the desired cyclization which accounts for the low chemical yields of 2-oxazoline obtained. Fujita and co-workers discovered that 2-iodo-1,3-dimethoxybenzene 9 can combine with its oxidized form, i.e. 2-(diacetoxyiodo)-1,3-dimethoxybenzene, under acidic conditions to generate a diaryliodonium salt; this side-reaction was proposed to be the cause of the relatively low yield of product using 9 as a pre-catalyst in an oxylactonization reaction. ${ }^{22}$

Scheme 5. Monitoring of the oxidation of iodoarenes by ${ }^{1} \mathrm{H}$ NMR 


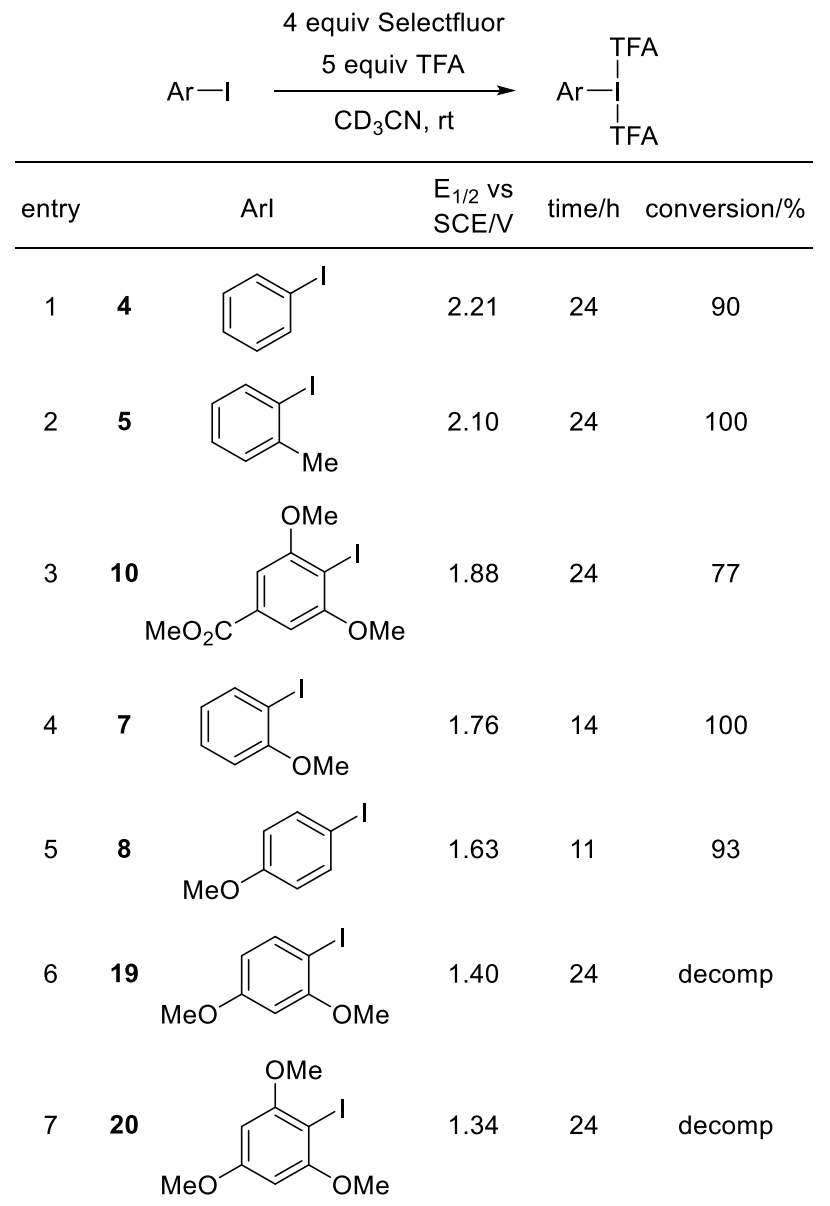

Minor modifications to 2-iodoanisole 7 (still our optimal precatalyst) could be investigated to identify a slightly more efficient catalytic system, however these analogs would probably not be commercially available and, so, would be of little impact. 1-Iodo-2,4-dimethoxybenzene 19 could be a highly efficient precatalyst in other hypervalent iodine mediated processes that occur on a faster timescale than its decomposition; however, not being able to recover the iodoarene is a disadvantage.

Interestingly, the experimentally derived initial rate of cyclization catalyzed by iodoarene $\mathbf{1 0}$ matches well with the calculated oxidation potential and HOMO energy but not with the calculated activation energy value (vide supra). This suggests that there was a change in the rate determining 
step with this iodoarene from the cyclization event to the iodoarene oxidation. Indeed, the rate of oxidation of 10 was comparable with that of iodobenzene 4.

Displacement of Iodoarene by Ligand Coupling or $S_{\mathbf{N}} 2$ Displacement. In our original report, cyclization of cis-alkene $\mathbf{2 1}$ was reported to lead to formation of product $\mathbf{2}$ as only one diastereomer; with the relative stereochemistry being assigned by NMR analysis. ${ }^{9}$ Applying the calculated mechanism for $N$-allylamide 1 to the cyclization of 21 suggested that the all-syn diastereomer $\mathbf{2 3}$ would be formed rather than 22. It was suspected that a change in mechanism was responsible for the observed stereochemistry of $\mathbf{2 2}$ due to ligand coupling being favored over $\mathrm{S}_{\mathrm{N}} 2$ displacement. This $\mathrm{S}_{\mathrm{N}} 2$ process was more difficult in this case because of the increased sterics at the secondary carbon atom bonded to the iodane compared to the primary carbon in $\mathbf{1 7 .}$ In order to confirm this hypothesis, the cyclization of $\mathbf{2 4}$ was calculated using 2-iodoanisole $\mathbf{7}$ as the pre-catalyst (Scheme 6). To reduce the computational resource required, the methyl amide $\mathbf{2 4}$ was modelled rather than the 4-methoxyphenyl amide $\mathbf{2 1}$ as this was not envisaged to impact on the stereochemistry determining step. 
Scheme 6. Issues of diastereoselectivity

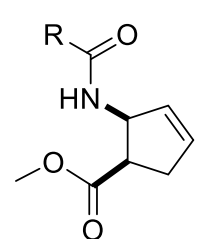

21

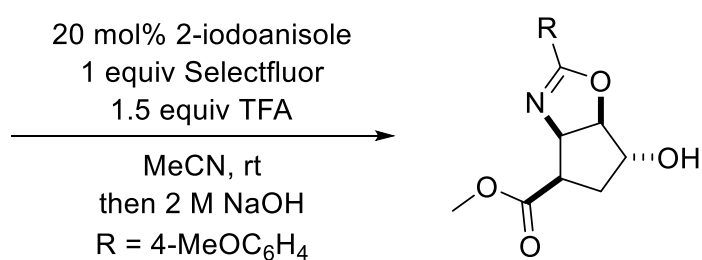

22 observed

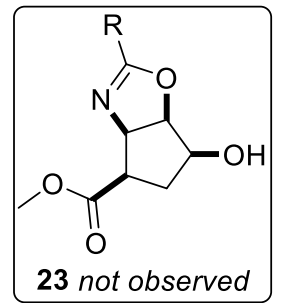

TS[27-29]
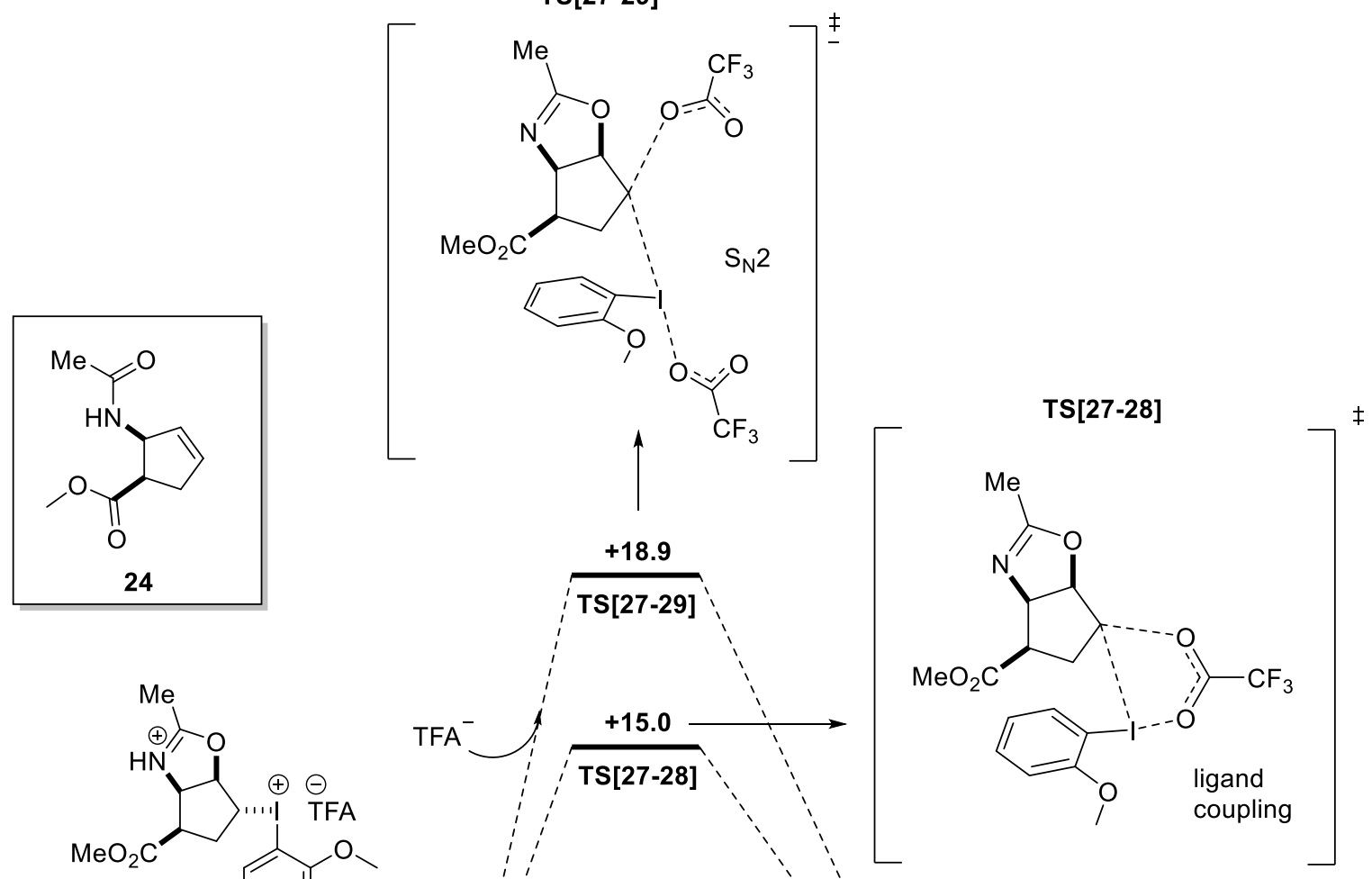

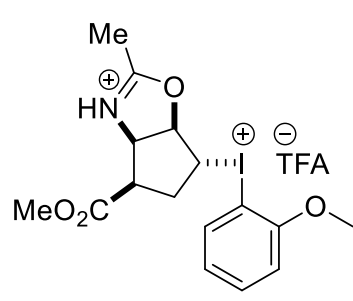

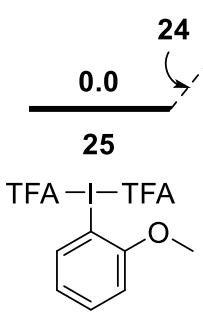

+1.3 TFA
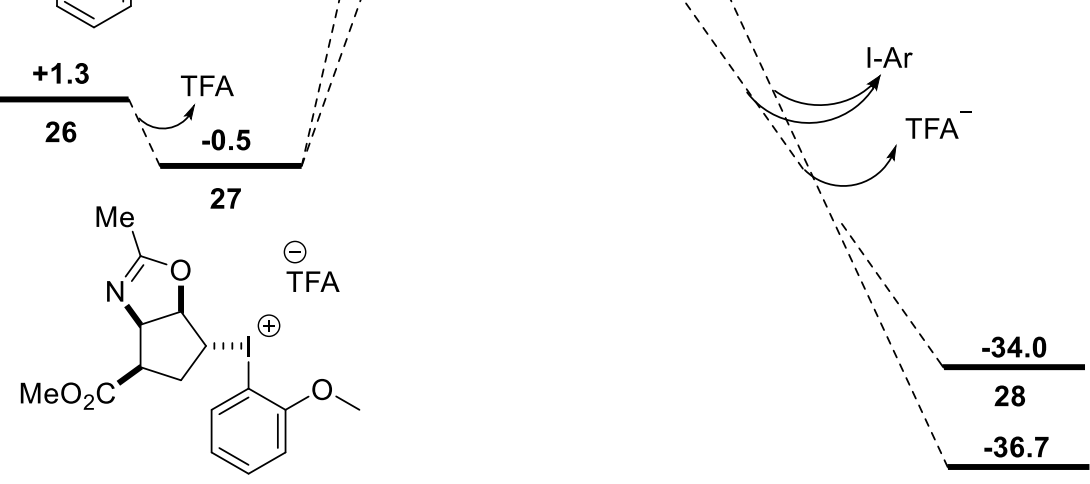

29
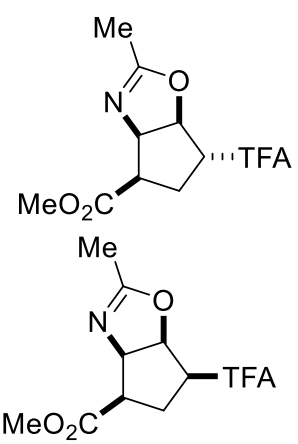
The free energies of the cyclized structures $\mathbf{2 6}$ and $\mathbf{2 7}$ resulting from trans-addition to the alkene compare favorably with the energies of the corresponding structures $\mathbf{1 6}$ and $\mathbf{1 7}$ for the cyclization of $N$-allylamide 1. As previously observed, reaction pathways proceeding through cis-additions could not be found. Conversion of iodane $\mathbf{2 7}$ to the final product could be achieved through either $\mathrm{S}_{\mathrm{N}} 2$ displacement or ligand coupling, but, in contrast to $\mathrm{N}$-allylamide, the latter pathway was favored by $3.9 \mathrm{kcal} \mathrm{mol}^{-1}$ resulting in a highly diastereoselective reaction. This result corroborated our stereochemical assignment of the product 22 and confirmed our hypothesis. Note that the allsyn diastereomer $\mathbf{2 9}$ is thermodynamically more stable than $\mathbf{2 8}$ but the reaction is under kinetic control. The optimized geometries are shown in Figure 5.

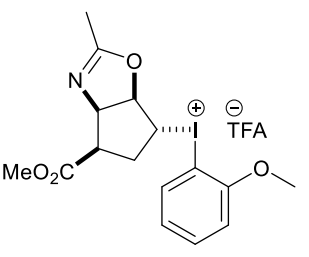

27

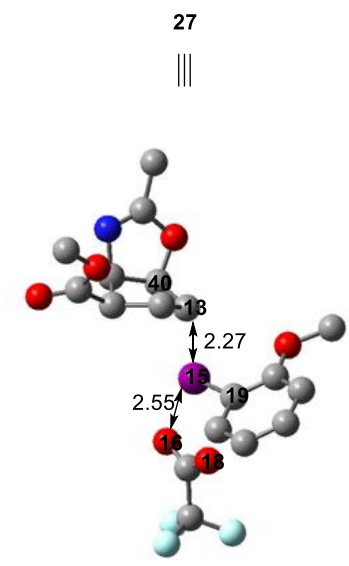

Angle C40-C13-I15 $=107.8^{\circ}$ Dihedral angle C40-C13-I15-C19 $=-179.9^{\circ}$

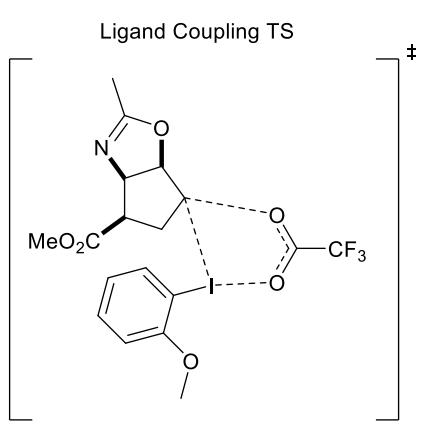

TS[27-28]

||

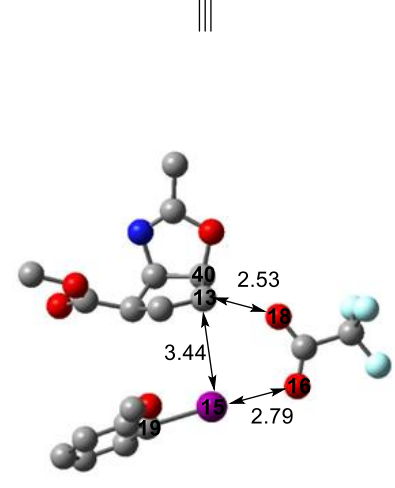

Angle C40-C13-I15 $=110.3^{\circ}$ Dihedral angle C40-C13-I15-C19 $=-98.6^{\circ}$

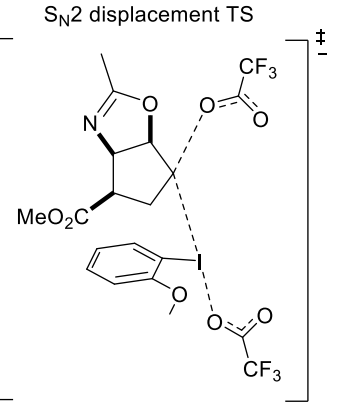

TS[27-29]

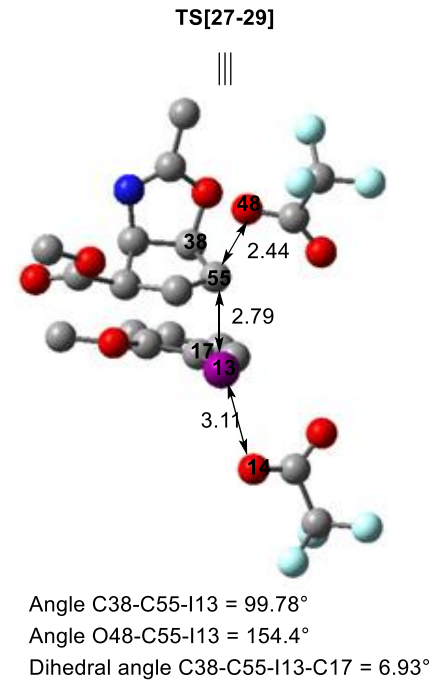

Figure 5. Optimized geometries of intermediate 27 and the ligand coupling and $\mathrm{S}_{\mathrm{N}} 2$ transition states. 


\section{CONCLUSION}

An in-depth analysis of the iodoarene-catalyzed cyclization of $N$-allylbenzamide $\mathbf{1}$ has been conducted using a combination of computational and kinetic experiments. A mechanism has been delineated which accounts for the experimental differences in rate observed with different iodoarene precatalysts and, importantly, the calculated free energy values correlate with our experimental results. The cyclization event has been shown to be rate-limiting and more electronrich iodoarenes lead to lower $\Delta G^{\ddagger}$ values for this step. The reaction has been found to be zero order in the precatalyst 2-iodoanisole 7 but the reaction model breaks down with more electron-rich iodoarenes due to competitive decomposition. The optimal choice of precatalyst for related processes is likely to be affected by the rate of the desired reaction; i.e. if the rate of reaction is faster than decomposition then a more electron-rich precatalyst such as 1-iodo-2,4dimethoxybenzene 19 could be the best option.

Loss of iodoarene from the reaction intermediate can occur by either $\mathrm{S}_{\mathrm{N}} 2$ displacement by a nucleophile (reductive elimination) or by ligand coupling at the iodine(III) center. Both pathways are possible but $\mathrm{S}_{\mathrm{N}} 2$ displacement is favored at primary carbons whereas ligand coupling is preferred with secondary carbons.

\section{EXPERIMENTAL SECTION}

General. ${ }^{1} \mathrm{H}$ NMR spectra were recorded in $\mathrm{ppm}$ from tetramethylsilane with the solvent resonance as the internal standard. All purchased reagents were used as received without further purification. Compounds $\mathbf{1},{ }^{23} \mathbf{1 0}^{24}$ and $\mathbf{2 0}^{25}$ were prepared by the literature procedures and the characterization data matched the reported values. 


\section{General Procedures for Kinetic Experiments}

Rate Data: Amide 1 (1 equiv) was added to a borosilicate NMR tube along with the iodoarene precatalyst ( 0.2 equiv), Selectfluor (1.5 equiv), TFA (1.5 equiv) and $\mathrm{CD}_{3} \mathrm{CN}$ ( $1 \mathrm{~mL}$ ) to generate a $0.3 \mathrm{M}$ solution of $\mathbf{1}$. The reaction mixture was monitored and the conversion of amide $\mathbf{1}$ was recorded by ${ }^{1} \mathrm{H}$ NMR spectroscopy (400 MHz, $298 \mathrm{~K}$ ) for up to 24 hours at $25{ }^{\circ} \mathrm{C}$. Assuming the reaction is first order in $\mathbf{1}$, the rate constant was determined by linearization of the decay curve followed by linear fitting for only the initial part of the reaction. Results are summarized in the supporting information table S1.

Effect of Catalyst Loading: The order of the cyclization reaction with respect to 2-iodoanisole was determined by the Variable Time Normalization Analysis (VTNA) as described by Burés (Scheme 3). ${ }^{14}$ Amide 1 (1 equiv) was added to a borosilicate NMR tube along with 2-iodoanisole (10 \& 30 mol\%), Selectfluor (1.5 equiv), TFA (1.5 equiv) and $\mathrm{CD}_{3} \mathrm{CN}$ ( $1 \mathrm{~mL}$ ). The reaction mixture was monitored by ${ }^{1} \mathrm{H}$ NMR spectroscopy (400 MHz, $298 \mathrm{~K}$ ) to study the effect of different catalyst loadings. The concentration of $\mathbf{1}$ was recorded for a period of 40 hours and plotted against a normalized time scale $t[\text { cat }]_{\mathrm{T}}{ }^{n}$; calculated by multiplying each time point by the total concentration of catalyst used in each experiment raised to an arbitrary power. The power value was adjusted to be zero as that produced the overlay of the curves (Scheme 3). The numerical data, along with the concentration of $\mathbf{1}$ at specific times, are listed in the supporting information.

Computational Methods: Quantum chemical calculations were performed using Gaussian 09, ${ }^{26}$ and Gauss View was used for molecular modelling. ${ }^{27}$ The geometry optimizations were performed in the gas phase under standard conditions using density functional theory $(\mathrm{DFT})^{28,29,30}$ in combination with the $6-31+\mathrm{G}(\mathrm{d}, \mathrm{p})^{31}$ basis set for all atoms except iodine, for 
which the $\mathrm{SDD}^{32}$ (Stuttgart/Dresden) effective core potential was used. All the bond lengths are given in Angstroms. Vibrational frequency calculations were performed in order to determine the imaginary frequencies for the respective molecules. The imaginary frequencies verified whether the stationary points are minima, having no imaginary frequencies, or transition states, possessing one imaginary frequency. The connectivity of the transition states was confirmed by computing IRC (intrinsic reaction coordinate) from the transition-state geometry towards both the reactant and product. ${ }^{33,34}$

Solvent effects were taken into account using the conductor-like polarizable continuum model (CPCM) ${ }^{35}$ Single-point calculations on the gas-phase optimized geometries were performed to estimate the change in energy in the presence of the solvent, acetonitrile. The triple-zeta quality $6-311++\mathrm{G}(\mathrm{d}, \mathrm{p})$ basis set along with SDD for I was used to account for the solvent effects. The Gibbs free energy values provided in the text are Gibbs energy in solution, $G_{s o l n}$, calculated by using the equation $G_{\text {sol }}=E_{\text {solv }}+G_{\text {nes }}+\Delta G_{\text {corr_gas. }}{ }^{36}$ The sums of the electronic and thermal free energies $(G)$ for reactants and transition states were obtained by the standard procedure in the framework of the harmonic approximation. ${ }^{37}$ The $\Delta G^{\dagger}$ of the reactions were calculated from the differences in the $G$ values of the transition states and the reactants.

\section{ASSOCIATED CONTENT}

\section{Supporting Information}

The following files are available free of charge.

Kinetic data and computational details, including Cartesian coordinates and energies (pdf). 


\section{AUTHOR INFORMATION}

\section{Corresponding Author}

*E-mail: w.j.moran@hud.ac.uk

ORCID

Wesley J. Moran: 0000-0002-5768-3629

Smaher Butt: 0000-0001-9861-5924

\section{Author Contributions}

The manuscript was written through contributions of all authors. All authors have given approval to the final version of the manuscript.

\section{Notes}

The authors declare no competing financial interests.

\section{ACKNOWLEDGMENT}

We thank the University of Huddersfield for a postgraduate scholarship (SEB) and the Université de Pau et des Pays de l'Adour for access to their computer cluster. We appreciate useful discussions with Professor Andy Laws (University of Huddersfield).

\section{REFERENCES}

${ }^{1}$ Selected reviews on hypervalent iodine chemistry: (a) Grelier, G.; Darses, B.; Dauban, P. Hypervalent organoiodine compounds: from reagents to valuable building blocks in synthesis. Beilstein J. Org. Chem. 2018, 14, 1508-1528. (b) Yoshimura, A.; Zhdankin, V. V. Advances in 
Synthetic Applications of Hypervalent Iodine Compounds. Chem. Rev. 2016, 116, 3328-3435. (c) Zheng, Z. S.; Zhang-Negrerie, D.; Du, Y. F.; Zhao, K. The applications of hypervalent iodine(III) reagents in the constructions of heterocyclic compounds through oxidative coupling reactions. Sci. China Chem. 2014, 57, 189-214. (d) Zhdankin, V. V. Hypervalent Iodine Chemistry: Preparation, Structure, and Synthetic Applications of Polyvalent Iodine Compounds; Wiley: New York, 2013.

${ }^{2}$ Selected reviews on hypervalent iodine catalysis: (a) Yusubov, M. S.; Zhdankin, V. V. Iodine catalysis: A green alternative to transition metals in organic chemistry and technology. Res.-Effic. Technol. 2015, 1, 49-67. (b) Yusubov, M. S.; Zhdankin, V. V. Development of new recyclable reagents and catalytic systems based on hypervalent iodine compounds. Mendeleev Commun. 2010, 20, 185-191. (c) Dohi, T.; Kita, Y. Hypervalent iodine reagents as a new entrance to organocatalysts. Chem. Commun. 2009, 2073-2085.

${ }^{3}$ For a recent example and discussion of this, see: Antien, K.; Pouységu, L. Deffieux, D.; Massip, S.; Peixoto, P. A.; Quideau, S. Synthesis of [7]Helicene Enantiomers and Exploratory Study of Their Conversion into Helically Chiral Iodoarenes and Iodanes. Chem. Eur. J. 2019, 25, 28522858.

${ }^{4}$ (a) Fuchigami, T.; Fujita, T. Electrolytic Partial Fluorination of Organic Compounds. 14. The First Electrosynthesis of Hypervalent Iodobenzene Difluoride Derivatives and Its Application to Indirect Anodic gem-Difluorination. J. Org. Chem. 1994, 59, 7190-7192. (b) Fuchigami, T.; Fujita, T. Electrolytic Partial Fluorination of Organic Compounds. 20. Electrosynthesis of Novel Hypervalent Iodobenzene Chlorofluoride Derivatives and Its Application to Indirect Anodic gemDifluorination. Tetrahedron Lett. 1996, 37, 4725-4728. 
${ }^{5}$ Ochiai, M.; Takeuchi, Y.; Katayama, T.; Sueda, T.; Miyamoto, K. Iodobenzene-catalyzed $\alpha-$ acetoxylation of ketones. In situ generation of hypervalent (diacyloxyiodo)benzenes using $m$ chloroperbenzoic acid. J. Am. Chem. Soc. 2005, 127, 12244-12245.

${ }^{6}$ Dohi, T.; Maruyama, A.; Yoshimura, M.; Morimoto, K.; Tohma, H.; Kita, Y. Versatile Hypervalent-Iodine(III)-Catalyzed Oxidations with $m$-Chloroperbenzoic Acid as a Cooxidant. Angew. Chem. Int. Ed. 2005, 44, 6193-6196.

${ }^{7}$ Zhong, W.; Liu, S.; Yang, J.; Meng, X.; Li, Z. Metal-Free, Organocatalytic Syn Diacetoxylation of Alkenes. Org. Lett. 2012, 14, 3336-3339.

${ }^{8}$ Yoshimura, A.; Middleton, K. R.; Todora, A. D.; Kastern, B. J.; Koski, S. R.; Maskaev, A. V.; Zhdankin, V. V. Hypervalent Iodine Catalyzed Generation of Nitrile Oxides from Oximes and their Cycloaddition with Alkenes or Alkynes. Org. Lett. 2013, 15, 4010.

${ }^{9}$ Zhao, Z.; Britt, L. H.; Murphy, G. K. Oxidative, Iodoarene-Catalyzed Intramolecular Alkene Arylation for the Synthesis of Polycyclic Aromatic Hydrocarbons. Chem. Eur. J. 2018, 24, 1700217005.

${ }^{10}$ Alhalib, A.; Kamouka, S.; Moran, W. J. Iodoarene-Catalyzed Cyclizations of Unsaturated Amides. Org. Lett. 2015, 17, 1453-1456.

${ }^{11}$ Kamouka, S.; Moran, W. J. Iodoarene-catalyzed cyclizations of $N$-propargylamides and $\gamma$ amidoketones: synthesis of 2-oxazolines. Beilstein J. Org. Chem. 2017, 13, 1823-1827.

12 Sreenithya, A.; Surya, K.; Sunoj, R. B. Hypercoordinate Iodine(III) Promoted Reaction and Catalysis: An Update on Current Mechanistic Understanding. WIREs Comput. Mol. Sci. 2017, 7, e1299. 10.1002/wcms. 1299. 
${ }^{13}$ Selected examples include: (a) Ganji, B.; Ariafard, A. DFT Mechanistic Investigation Into Phenol Dearomatization Mediated by an Iodine(III) Reagent. Org. Biomol. Chem. 2019, 17, 35213528. (b) Zhou, L.; Li, S. Charge - Transfer Induced para-Selective $\mathrm{sp}^{2} \mathrm{C}-\mathrm{H}$ Bond Activation of Arenes by Use of a Hypervalent Iodine Compound: A Theoretical Study. J. Org. Chem. 2017, 82, 2984-2991. (c) Beaulieu, S.; Legault, C. Y. Mechanistic Insights on the Iodine(III)-Mediated $\alpha$ Oxidation of Ketones. Chem. Eur. J. 2015, 21, 11206-11211.

${ }^{14}$ (a) Zhou, B.; Haj, M. K.; Jacobsen, E. N.; Houk, K. N.; Xue, X.-S. Mechanism and Origins of Chemo- and Stereoselectivities of Aryl Iodide-Catalyzed Asymmetric Difluorinations of $\beta$ Substituted Styrenes. J. Am. Chem. Soc. 2018, 140, 15206-15218. Also, see: (b) Zheng, H,; Sang, Y.; Houk, K. N.; Xue, X.-S., Cheng, J.-P. Mechanism and Origins of Enantioselectivities in Spirobiindane-Based Hypervalent Iodine(III)-Induced Asymmetric Dearomatizing Spirolactonizations. J. Am. Chem. Soc. 2019, 141, 16046-16056.

${ }^{15}$ Burés, J. A Simple Graphical Method to Determine the Order in Catalyst. Angew. Chem. Int. Ed. 2016, 55, 2028-2031.

16 Toby, S. Does a Photochemical Reaction Have a Kinetic Order? J. Chem. Educ. 2005, 82, 3738.

${ }^{17}$ Lin, S. H.; Eyring, H. Kinetics of Heterogeneous Chemical Reactions, II. Proc. Nat. Acad. Sci. 1970, 65, 47-50.

18 Selected examples include: (a) Fujita, M.; Ookubo, Y.; Sugimura, T. Asymmetric cycloetherification based on a chiral auxiliary for 4-acyloxy-1-butene substrates during oxidation with iodosylbenzene via a 1,3-dioxan-2-yl cation. Tetrahedron Lett. 2009, 50, 1298-1300. (b) Lovick, H. M.; Michael, F. E. Metal-Free Highly Regioselective Aminotrifluoroacetoxylation of 
Alkenes. J. Am. Chem. Soc. 2010, 132, 1249-1251. (c) Saito, A.; Matsumoto, A.; Hanzawa, Y. PIDA-mediated synthesis of oxazoles through oxidative cycloisomerization of propargylamides. Tetrahedron Lett. 2010, 51, 2247-2250. (d) Zhou, Z.-S.; He, X.-H. A convenient phosphoryloxylactonization of pentenoic acids with catalytic hypervalent iodine(III) reagent. Tetrahedron Lett. 2010, 51, 2480-2482.

19 Experimental work confirmed that the cyclization reaction occurred with pre-formed bis(trifluoroacetoxy)iodoarene without any fluorine sources present in the reaction mixture.

${ }^{20}$ Roth, H. G.; Romero. N. A.; Nicewicz, D. A. Experimental and Calculated Electrochemical Potentials of Common Organic Molecules for Applications to Single-Electron Redox Chemistry. Synlett 2016, 27, 714-723.

${ }^{21}$ Hamza, M.; Ammar, S.; Abdelhédi, R. Electrochemical oxidation of 1,3,5-trimethoxybenzene in aqueous solutions at gold oxide and lead dioxide electrodes. Electrochim. Acta 2011, 56, 37853789.

${ }^{22}$ Shimogaki, M.; Fujita, M.; Sugimura, T. Enantioselective Oxidation of Alkenylbenzoates Catalyzed by Chiral Hypervalent Iodine(III) To Yield 4-Hydroxyisochroman-1-ones. Eur. J. Org. Chem. 2013, 31, 7128-7138.

${ }^{23}$ Vyklický, L; Dvořáková, H; Dvořák, D. Iron Aminocarbene Complexes Containing a Double $\mathrm{C}=\mathrm{C}$ Bond in the $\mathrm{N}$-Substituent: Preparation and Reactivity. Organometallics 2001, 20, 5419 5424.

${ }^{24}$ Denmark, S. E; Regens, C. S; Kobayashi, T. Total Synthesis of Papulacandin D. J. Am. Chem. Soc. 2007, 10, 2774-2776. 
25 Heng, S; Harris, K. M.; Kantrowitra, E. R. Designing Inhibitors Against Fructose 1,6bisphosphatase: Exploring Natural Products for Novel Inhibitor Scaffolds. Eur. J. Med. Chem. 2010, 45, 1478-1484.

${ }^{26}$ Frisch, M. J.; Trucks, G. W.; Schlegel, H. B.; Scuseria, G. E.; Robb, M. A.; Cheeseman, J. R.; Scalmani, G.; Barone, V.; Mennucci, B.; Petersson, G. A.; Nakatsuji, H.; Caricato, M.; Li, X.; Hratchian, H. P.; Izmaylov, A. F.; Bloino, J.; Zheng, G.; Sonnenberg, J. L.; Hada, M.; Ehara, M.; Toyota, K.; Fukuda, R.; Hasegawa, J.; Ishida, M.; Nakajima, T.; Honda, Y.; Kitao, O.; Nakai, H.; Vreven, T.; Montgomery, J. A., Jr.; Peralta, J. E.; Ogliaro, F.; Bearpark, M.; Heyd, J. J.; Brothers, E.; Kudin, K. N.; Staroverov, V. N.; Kobayashi, R.; Normand, J.; Raghavachari, K.; Rendell, A.; Burant, J. C.; Iyengar, S. S.; Tomasi, J.; Cossi, M.; Rega, N.; Millam, J. M.; Klene, M.; Knox, J. E.; Cross, J. B.; Bakken, V.; Adamo, C.; Jaramillo, J.; Gomperts, R.; Stratmann, R. E.; Yazyev, O.; Austin, A. J.; Cammi, R.; Pomelli, C.; Ochterski, J. W.; Martin, R. L.; Morokuma, K.; Zakrzewski, V. G.; Voth, G. A.; Salvador, P.; Dannenberg, J. J.; Dapprich, S.; Daniels, A. D.; Farkas, O.; Foresman, J. B.; Ortiz, J. V.; Cioslowski, J.; Fox, D. J. Gaussian 09, revision D.01; Gaussian Inc.: Wallingford, CT, 2009.

${ }^{27}$ Gauss View, Version 5, Dennington, Roy; Keith, Todd A.; Millam, John M. Semichem Inc., Shawnee Mission, KS, 2016.

${ }^{28}$ Becke, A. D. A new mixing of Hartree-Fock and Local Density Functional Theories. J. Chem. Phys. 1993, 98, 1372-1377.

${ }^{29}$ Becke, A. D. Density-Functional Exchange-Energy Approximation with Correct Asymptotic Behavior. Phys. Rev. A. 1988, 38, 3098. 
${ }^{30}$ Lee, C.; Yang, W.; Parr, R. G. Development of the Colle-Salvetti Correlation-Energy Formula into a Functional of the Electron Density. Phys. Rev. B: Condens. Matter 1988, 37, 785.

${ }^{31}$ Hariharan, P. C.; Pople, J.A. The Influence of Polarization Functions on Molecular Orbital Hydrogenation Energies. Theoret. Chim. Acta 1973, 28, 213-222.

32 Igel-Mann, G.; Stoll, H., Preuss, H. Pseudopotentials For Main Group Elements (IIIa through VIIa), Mol. Phys. 1988, 65, 1321-1328.

${ }^{33}$ Gonzalez, C.; Schlegel, H. B. An Improved Algorithm for Reaction Path Following. J. Chem. Phys. 1989, 90, 2154-2161.

${ }^{34}$ Gonzalez, C.; Schlegel, H. B. Reaction path following in mass-weighted internal coordinates. J. Phys. Chem. 1990, 94, 5523-5527.

${ }^{35}$ Cossi, M.; Rega, N.; Scalmani, G.; Barone, V. Energies, Structures, and Electronic Properties of Molecules in Solution with the C-PCM solvation Model. J. Comput. Chem. 2003, 24, 669-681.

${ }^{36}$ Ho, J.; Klamt, A.; Coote, M. L. Comment on the Correct Use of Continuum Solvent Models. J. Phys. Chem. A. 2010, 114, 13442-13444.

37 McQuarrie, D.; Simon, J. D. Molecular Thermodynamics. University Science Books, Sausalito, CA, 1999. 